\title{
Modulation instability, Fermi-Pasta-Ulam recurrence, rogue waves, nonlinear phase shift, and exact solutions of the Ablowitz-Ladik equation
}

\author{
Nail Akhmediev and Adrian Ankiewicz \\ Optical Sciences Group, Research School of Physics and Engineering, Institute of Advanced Studies, The Australian National University, \\ Canberra ACT 0200, Australia
}

(Received 16 August 2010; revised manuscript received 18 February 2011; published 20 April 2011)

\begin{abstract}
We study modulation instability (MI) of the discrete constant-background wave of the Ablowitz-Ladik (A-L) equation. We derive exact solutions of the A-L equation which are nonlinear continuations of MI at longer times. These periodic solutions comprise a family of two-parameter solutions with an arbitrary background field and a frequency of initial perturbation. The solutions are recurrent, since they return the field state to the original constant background solution after the process of nonlinear evolution has passed. These solutions can be considered as a complete resolution of the Fermi-Pasta-Ulam paradox for the A-L system. One remarkable consequence of the recurrent evolution is the nonlinear phase shift gained by the constant background wave after the process. A particular case of this family is the rational solution of the first-order or fundamental rogue wave.

DOI: 10.1103/PhysRevE.83.046603

PACS number(s): 05.45.Yv, 45.05.+x, 02.30.Ik
\end{abstract}

\section{INTRODUCTION}

Modulation instability (MI) is a universal phenomenon that occurs in many physical systems. It has been studied in optics [1], hydrodynamics [2], and even in biology [3]. In the ocean, modulation instability may cause the inception of the infamous rogue waves [4]. It is less commonly known that a developed stage of modulation instability can result in breather-type solutions that are presently known as "Akhmediev breathers" (ABs) [5-9]. Remarkably, modulation instability exists not only in continuous media but can develop in discrete systems, such as those described by the Ablowitz-Ladik (A-L) equation [10]. A discrete electrical lattice employing inductors and nonlinear capacitors in a transmission line is a relevant realization described by the A-L and related equations [11]. The authors of Ref. [11] have presented regions of MI for this case, while energy localization via MI has been studied in Ref. [12].

There are many publications relating to the A-L equation and discrete systems in general. These include a review article [13] on wave transmission in nonlinear lattices. In particular, it gives an overview of the A-L equation and other discretizations of the nonlinear Schrödinger equation (NLSE), with applications to optical and electrical systems. One of the basic questions that has not been answered yet is: In discrete systems, does MI result in breather-type solutions? We show, in this work, that indeed, exact solutions starting with modulation instability do exist, and we present explicit forms for them. Our preliminary studies have been presented in Ref. [14] and here we give a more detailed answer to the above question. Interestingly, these solutions have the property of recurrence in the Fermi-Pasta-Ulam (FPU) sense [15], i.e., on starting from a ground state of homogeneous background, they eventually return the system to the same state of homogeneous background, apart from a nonlinear shift in phase. This phase shift is, in a sense, analogous to the Berry phase shift [16], but is a specific property of nonlinear systems. It was first discovered for the NLSE in the work of Ref. [17]. We show here that the breather solution of the A-L equation also causes the background state to shift its phase nonlinearly when the localized formation defined by it has passed.
A specific feature of our derivation of the A-L breather solution here is a special ansatz that has been suggested in Ref. [17] for the NLSE. Namely, real and imaginary parts of the solution are coupled by a linear relation. We use the same ansatz for the A-L equation. Despite the fact that the A-L system is a very special discretization of the NLSE, a similar ansatz proves to be useful, as it allows us to find the breather-type exact solution.

The standard form of the integrable A-L equation can be written as $[18,19]$

$$
i \frac{\partial \psi_{n}}{\partial t}+\left(\psi_{n-1}+\psi_{n+1}\right)\left(1+\left|\psi_{n}\right|^{2}\right)-2 \psi_{n}=0,
$$

where $t$ is the continuous evolution variable (time or longitudinal spatial variable) and $n=0, \pm 1, \pm 2, \ldots$ are integers. In contrast to the original NLSE, this discretization has a nonlinear term which is nonlocal. Thus, we cannot directly discretize the solutions that have been obtained for the NLSE. However, the A-L equation is also integrable, thus providing a chance of deriving discrete solutions which are similar to solutions of the NLSE. In Sec. II, we give a perturbation solution, which is only valid for the initial growth of a small ripple on the cw background. However, the results in Secs. III-X are all exact solutions of the A-L equation.

\section{MODULATION INSTABILITY}

First, we will present results for modulation instability of the A-L equation. Namely, we wish to find the range of spatial frequencies which can grow due to MI on a "constant background" (CB) solution. This solution is

$$
\psi_{n}=-q \exp \left(i 2 q^{2} t+i \phi\right),
$$

where $q$ is the real background amplitude with the corresponding propagation constant $2 q^{2}$. The wave can have arbitrary phase $\phi$, while the minus sign is written here for convenience.

To eliminate fast oscillations in $t$, we can work with the functions $\Psi_{n}(t)$ such that

$$
\psi_{n}(t)=\Psi_{n}(t) \exp \left(i 2 q^{2} t\right) .
$$


Then this function satisfies the modified A-L equation

$$
i \frac{\partial \Psi_{n}}{\partial t}+\left(\Psi_{n-1}+\Psi_{n+1}\right)\left(1+\left|\Psi_{n}\right|^{2}\right)-2\left(q^{2}+1\right) \Psi_{n}=0,
$$

If we know one set of functions, say, $\Psi_{n}(t)$, it is easy to transform it to the original set $\psi_{n}(t)$ and vice versa.

Suppose we apply a small periodic perturbation to the CB. Then the approximate solution the of A-L equation, Eq. (3), can be written in the form

$$
\Psi_{n}(t)=\{-q+[a(t)+i b(t)] \cos (\kappa n)\} \exp (i \phi),
$$

We assume that $a(t)$ and $b(t)$ are small real functions and that $\kappa$ is the transverse frequency of perturbation. Although we take the perturbation in the form of a cosine function, it is defined on a discrete set of points. Thus, strictly speaking, the perturbation is not periodic. It is periodic only when the period $T=2 \pi / \kappa$ is an integer. However, the functions we are dealing with can be continuously shifted, i.e., the discrete variable $n$ can be replaced by $n-n_{0}$, with $n_{0}$ being a real noninteger parameter. Solutions remain valid for any real $n_{0}$. This means that, to some extent, we can operate with the variable $n$ as a continuous one, and thus we deal with periodic functions.

Substituting Eq. (4) into the A-L equation, Eq. (3), leads to a set of coupled differential equations:

$$
\begin{gathered}
a^{\prime}(t)=4\left(1+q^{2}\right) b(t) \sin ^{2}\left(\frac{\kappa}{2}\right) \\
b^{\prime}(t)=2\left[q^{2}-1+\left(q^{2}+1\right) \cos (\kappa)\right] a(t) .
\end{gathered}
$$

Eliminating $a(t)$ produces the second-order equation

$$
b^{\prime \prime}(t)=\delta^{2} b(t),
$$

which has an exponentially growing or decaying solution $b(t)=b_{0} \exp ( \pm \delta t)$ with real $\delta$ which can be found from

$$
\delta^{2}=8\left(1+q^{2}\right) \sin ^{2}\left(\frac{\kappa}{2}\right)\left[q^{2}-1+\left(q^{2}+1\right) \cos (\kappa)\right] .
$$

Simplifying this equation, we find

$$
\delta=2 \sqrt{q^{4}-\left[\left(1+q^{2}\right) \cos (\kappa)-1\right]^{2}} .
$$

Of course, $-\delta$ is equally valid. Solutions of Eq. (7) can be a linear combination of exponential functions,

$$
b(t)=b_{01} \exp (+\delta t)+b_{02} \exp (-\delta t)
$$

or linear combination of hyperbolic functions

$$
b(t)=b_{03} \cosh (\delta t)+b_{04} \sinh (\delta t),
$$

with small real constants $b_{0 i}$. This shows that the $\mathrm{CB}$ wave solution, Eq. (2), or

$$
\Psi_{n}=-q \exp (i \phi),
$$

is a saddle point of the infinite-dimensional dynamical system defined by the A-L equation. As it can have arbitrary phase $\phi$, any point on a circle around the origin on the complex plane with the radius $q$ is a saddle point. When either of the two constants $b_{01}$ or $b_{02}$ is zero, the trajectories start or finish at the saddle point, thus defining the beginning or the end of the homoclinic or heteroclinic orbit.

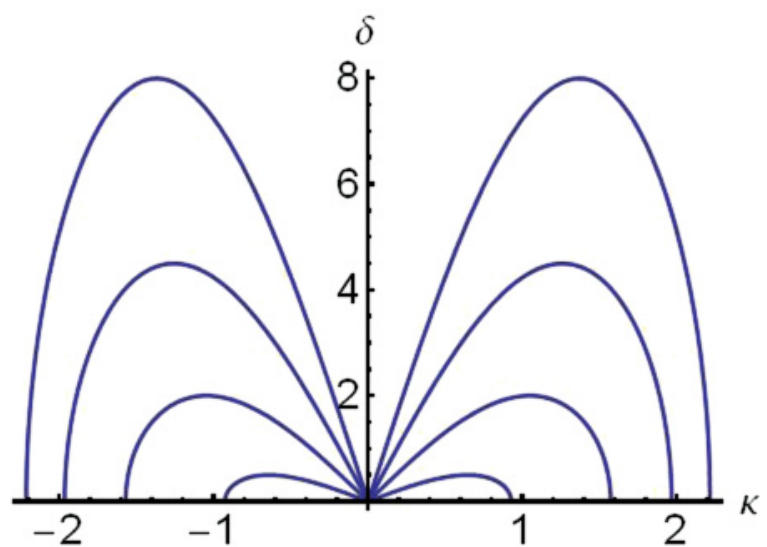

FIG. 1. (Color online) Growth rate of MI, $\delta$, defined by Eq. (9). From upper to lower, the curves are calculated for $q=2,1.5,1$, and 0.5 , respectively.

In Fig. 1, we plot $\delta$ (the growth rate), which is real and positive in the interval of allowable $\kappa$ values:

$$
0<\kappa<(\kappa)_{\max } \text {. }
$$

The upper limit of unstable frequencies,

$$
(\kappa)_{\max }=\arccos \left(\frac{1-q^{2}}{1+q^{2}}\right),
$$

depends on the CB amplitude $q$. Perturbations with frequencies within the instability band experience growth due to modulation instability. Depending on the relation between $a$ and $b$, they can equally well experience decay. The maximum value of the instability growth rate,

$$
\delta_{\max }=2 q^{2},
$$

also depends on the amplitude of the background $q$ and occurs at $\kappa=\arccos \left(\frac{1}{1+q^{2}}\right)$. Qualitatively, the curves of instability are similar to those obtained for the continuous NLSE (see Fig. (3.17) of Ref. [20]).

In order to eliminate trigonometric functions from $\delta$, we define

$$
v=\sin ^{2}\left(\frac{\kappa}{2}\right) .
$$

Then the MI growth rate, Eq. (9), can be written in the form

$$
\delta=4 \sqrt{\left(1+q^{2}\right) v\left[q^{2}(1-v)-v\right]} .
$$

\section{LINEAR ANSATZ}

Clearly, the solution Eq. (4) is approximate. Sometimes approximate solutions are used, with variational principles, etc. In that case there is a choice of various similar functions. However, this is not our approach here, and we stress that, henceforth, all solutions in the rest of this paper are exact. In order to find an exact solution that is a continuation of Eq. (4) when the perturbation increases, we use a special ansatz. The continuous NLS equation can be solved with a linear ansatz which was first used in the work of Ref. [17]. This ansatz leads to a rich family of first-order solutions of the NLSE [21]. Thus, a whole class of solutions can be derived from first principles. 
Our conjecture here is that the same ansatz can be used for the A-L equation, Eqs. (1) or (3). Generally, the complex function $\Psi_{n}(t)$ can be written explicitly as

$$
\Psi_{n}(t)=\left[R_{n}(t)+i J_{n}(t)\right] e^{i \phi},
$$

where $R_{n}(t)$ and $J_{n}(t)$ are real and imaginary parts of $\Psi_{n}$ without the phase factor. The exponential $e^{\phi}$ defines the initial phase of the CB. We propose that the real and imaginary parts of the solution in the complex plane are linearly related, i.e., for arbitrary $n$, we can write

$$
J_{n}(t)=m(t)\left[R_{n}(t)+q\right],
$$

where the coefficient $m(t)$ depends only on $t$. If this is the case, we can use Eqs. (13) and (14) in Eq. (3) and obtain exact solutions.

First, from Eq. (14), we have

$$
J_{n}^{\prime}(t)=m^{\prime}(t)\left(R_{n}(t)+q\right)+m(t) R_{n}^{\prime}(t) .
$$

Then, substituting Eqs. (14) and (15) into Eq. (3), we separate the real and imaginary parts of the A-L equation and, as a result, obtain two real equations,

$$
\begin{aligned}
& \left(R_{n-1}+R_{n+1}\right)\left[1+\left(R_{n}\right)^{2}+m^{2}\left(R_{n}+q\right)^{2}\right] \\
& \quad-2\left(1+q^{2}\right) R_{n}-m^{\prime}\left(R_{n}+q\right)-m R_{n}^{\prime}=0
\end{aligned}
$$

and

$$
\begin{aligned}
& m\left(R_{n-1}+R_{n+1}+2 q\right)\left[1+\left(R_{n}\right)^{2}+m^{2}\left(R_{n}+q\right)^{2}\right] \\
& \quad+R_{n}^{\prime}-2\left(1+q^{2}\right) m\left(R_{n}+q\right)=0 .
\end{aligned}
$$

Now, we substitute $R_{n-1}+R_{n+1}$ from Eq. (16) into Eq. (17) and get a first-order differential equation in $R_{n}(t)$. We simplify this further by defining

$$
P_{n}(t)=\frac{1}{R_{n}(t)+q} .
$$

Then we obtain

$$
P_{n}^{\prime}(t)=2 q m(t)+\frac{m(t)\left[m^{\prime}(t)-4 q^{2}\right]}{1+m^{2}(t)} P_{n}(t) .
$$

This can be regarded as a compatibility condition between Eqs. (16) and (17). Various simple forms of the slope $m(t)$ admit specific solutions of Eq. (19) and hence exact solutions of the A-L equation itself. However, $m(t)$ is not an arbitrary function.

\section{EXACT SOLUTIONS AS CONTINUATION OF MODULATION INSTABILITY}

First, we use a slope of the form

$$
m(t)=\frac{\delta}{4 v\left(1+q^{2}\right)} \tanh (\delta t)
$$

for arbitrary real background $q$ and real $v$. Its time dependence is similar to that used for the NLSE [17]. Clearly, the parameter $v$ has to be in the range $0<v<\frac{q^{2}}{1+q^{2}}$. Then, Eq. (19) can be written in explicit form.

The limit $m(\infty)$ is finite. Moreover, $m^{\prime}(\infty)=0$. Thus, $P_{n}(\infty)$ is also finite. We can see directly from Eq. (19) that

$$
P_{n}(\infty)=\frac{1}{2 q}\left[1+m^{2}(\infty)\right]=\frac{q}{2 v\left(1+q^{2}\right)} .
$$

Solving the differential equation (19) with the above condition at infinity gives

$$
P_{n}(t)=\frac{q \pm a(n) \operatorname{sech}(\delta t)}{2 v\left(1+q^{2}\right)} .
$$

This is valid because the function $a(n)$ does not involve $t$. When we now substitute Eq. (20) into Eqs. (16) and (17), we get difference equations for the function $a(n)$. It is easily seen that these are satisfied by

$$
a(n)=c_{1} \cos (\kappa n)=c_{1} \cos [2 n \arcsin (\sqrt{v})],
$$

where the constant $c_{1}$ is given by

$$
c_{1}=\sqrt{\frac{q^{2}(1-v)-v}{1-v}},
$$

and we have used Eq. (11), i.e., $\kappa=2 \arcsin (\sqrt{v})$, to write the solution in simple form.

Using $R_{n}(t)=1 / P_{n}(t)-q$ from Eqs. (18) and (14), after simple transformations we can derive the "breather" (if we may use this word in the broad sense) solution,

$$
\Psi_{n}(t)=\left[\frac{2\left(1+q^{2}\right) v \cosh (\delta t)+i \frac{\delta}{2} \sinh (\delta t)}{q \cosh (\delta t) \pm c_{1} \cos (\kappa n)}-q\right] e^{i \phi},
$$

where $\kappa$ belongs to the interval of instability, Eq. (10). For arbitrary coefficients, direct substitution into Eq. (1) shows that this solution is valid. This solution is a direct analog of solution (38) of the work of Ref. [21] derived for the NLSE. It describes the growth and decay of modulation with period $\kappa$ on a constant background field $q$.

The solution given by Eq. (21) starts (at $t=-\infty$ ) with a constant background field slightly perturbed by a periodic modulation. Due to the instability, the modulation increases until it reaches its maximum value (at $t=0$ ) and decreases symmetrically afterward until $t=\infty$. Mathematically speaking, this solution is a heteroclinic orbit in the infinite dimensional phase space of the dynamical system given by the A-L equation. The starting and ending saddle points of this orbit are generally different. We stress that the solution is a two-parameter family. It depends on two independent parameters, $\kappa$ and $q$. We also assume that the discrete variable $n$ is taken in general form $n-n_{0}$, thus allowing us to keep one more free parameter, $n_{0}$, in the solution. This parameter results in translations along $n$ axis when $n_{0}$ is an integer, but changes the profile of the solution when $n_{0}$ is not integer.

The solution (21) can be derived as one of the limiting cases of the family of Narita solutions [14]. However, we should note that Narita solutions are presented in a form that requires solution of a coupled set of transcendental equations [22]. The result is still complicated and has to be further simplified to obtain Eq. (21). The solution in the form of Eq. (21) is explicit and is directly comparable with the corresponding solution of the NLSE [21].

Fixing one of the parameters, we can additionally simplify the solution. In order to do that, we can choose a special value of one of the independent parameters. Namely, if for $\kappa$, we choose the point of the maximum growth rate, $\kappa_{0}=\arccos \left(\frac{1}{1+q^{2}}\right)$, then the dependent parameters will take 


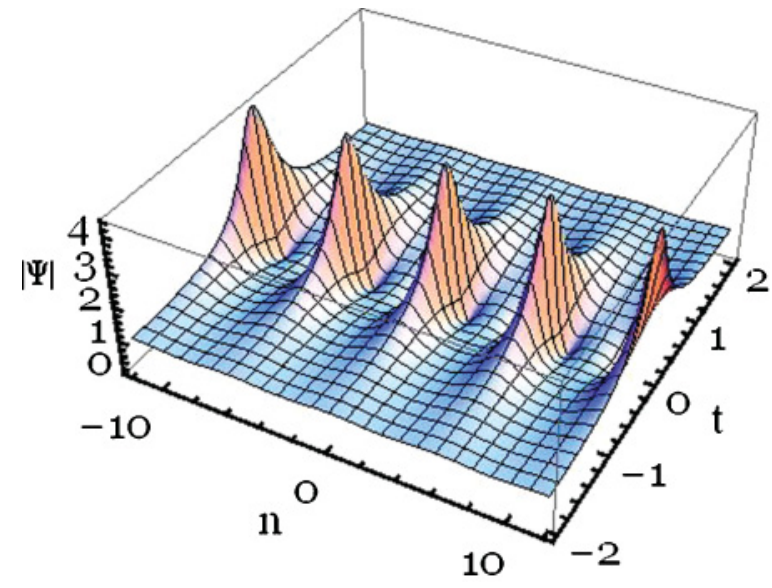

FIG. 2. (Color online) Breather solution of the A-L equation for maximum growth rate, given by Eq. (22). The evolution starts with a constant background, $q$, which is slightly modulated. Then the modulation increases to reach its maximum at $t=0$, and finally the solution returns to the original background, $q$, but with a nonlinear phase shift $\Delta \phi$. Here, $q=1$ and $\kappa=\pi / 3$, thus providing an integer period, $T=6$, along the $n$ axis. Additionally, we take $n_{0}=0$, thus locating the maxima of the solution at sites of the discrete set.

the following values: $\delta=2 q^{2}, v=\frac{q^{2}}{2\left(1+q^{2}\right)}$, and $c_{1}=q \sqrt{\frac{1+q^{2}}{2+q^{2}}}$. In this special case, the solution can be reduced to the form

$\Psi_{n}(t)=i q\left[\frac{\sqrt{2+q^{2}} \sinh \left(2 q^{2} t\right) \mp i \sqrt{1+q^{2}} \cos \left(\kappa_{0} n\right)}{\sqrt{2+q^{2}} \cosh \left(2 q^{2} t\right) \pm \sqrt{1+q^{2}} \cos \left(\kappa_{0} n\right)}\right] e^{i \phi}$,

where $\kappa_{0}$ is fixed, as given above. In this form, we have a complete analogy with the AB solution (11) of the work of Ref. [17]. This solution is illustrated in Fig. 2. Here, we have also fixed $q=1$. This choice provides an integer value for the period along the $n$ axis. When the shift $n_{0}$ is zero, the maximum values of the solution are located on discrete sites.

\section{CONSERVED QUANTITIES}

As the A-L equation is completely integrable, there is an infinite number of conserved quantities related to it. Here, we consider only the two lowest-order ones. However, as our solutions are on a constant background, the definitions are different from the standard case.

In fact, for localized solutions with zero background at infinity, i.e., when $n \rightarrow \pm \infty$, there is an "energy invariant" $\sum_{n=-\infty}^{\infty} Q_{n}$ that is conserved. If the background is not zero but has a finite value $q$, we need to redefine energy, since, with the standard definition, it would be infinite. Specifically, for a solution $\psi_{n}$, located on a background field $q$, we define [14]

$$
Q=\sum_{n=-\infty}^{\infty} Q_{n}
$$

where

$$
Q_{n}=\frac{1}{2}\left(\psi_{n} \psi_{n+1}^{*}+\psi_{n}^{*} \psi_{n+1}\right)-q^{2} .
$$

The redefined $Q$ is conserved, as can be seen from the simple considerations below.
When $T$ is an integer, the proof can be made analytically. For the important case of maximum growth rate, $q=1$ with $\kappa=\pi / 3$, we see that $T=6, v=1 / 4, \delta=2$, and $c_{1}=\sqrt{2 / 3}$, so we only need to add six terms in each case, since $\psi_{n+6}=\psi_{n}$, so $Q_{n+6}=Q_{n}$. Now, for $n_{0}=0$,

$$
\begin{gathered}
Q_{0}=Q_{5}=-\frac{3[2+\sqrt{6} \cosh (2 t)]}{5+3 \sqrt{6} \cosh (2 t)+3 \cosh (4 t)}, \\
Q_{1}=Q_{4}=-\frac{6}{2+3 \cosh (4 t)}, \\
Q_{2}=Q_{3}=\frac{3[-2+\sqrt{6} \cosh (2 t)]}{5-3 \sqrt{6} \cosh (2 t)+3 \cosh (4 t)} .
\end{gathered}
$$

Clearly $\sum_{n=0}^{5} Q_{n}=0$, so that the complete quantity $Q=0$, i.e., it is conserved.

When $T$ is not an integer, taking the sum analytically becomes cumbersome. However, numerical evaluations, even for a finite (but large) number of elements in the sum, Eq. (23), show that $Q$ does not depend on $t$ for the solution (21) with arbitrary coefficients.

The second conserved quantity is the momentum,

$$
M=\sum_{n=-\infty}^{\infty} M_{n}
$$

where $M_{n}=i\left(\psi_{n} \psi_{n+1}^{*}-\psi_{n}^{*} \psi_{n+1}\right)$. Thus $M_{n+6}=M_{n}$. In contrast to $Q$, the expression for the momentum is the same for solutions on zero or nonzero background. For the above case, $q=1$ and $\kappa=\pi / 3$ (again with $n_{0}=0$ ), we have

$$
\begin{gathered}
M_{5}=-M_{0}=-\frac{2 \sqrt{6} \sinh (2 t)}{5+3 \sqrt{6} \cosh (2 t)+3 \cosh (4 t)}, \\
M_{4}=-M_{1}=-\frac{4 \sqrt{6} \sinh (2 t)}{2+3 \cosh (4 t)}, \\
M_{3}=-M_{2}=-\frac{2 \sqrt{6} \sinh (2 t)}{5-3 \sqrt{6} \cosh (2 t)+3 \cosh (4 t)} .
\end{gathered}
$$

Simple calculations show that $\sum_{n=0}^{5} M_{n}=0$, thus leading to $M=0$. Thus, the momentum is also conserved. The derivation is valid for arbitrary values of the offset, $n_{0}$. For example, if $n_{0}=\frac{1}{2}$, then $M_{5}=-M_{1}$ and $M_{2}=-M_{4}$, while $M_{3}=-M_{0}=0$, so $M=0$. In fact, for arbitrary values of $q$ and $\kappa$, with $n_{0}=\frac{1}{2}$, the solution is not periodic, but we have

$$
M_{n}=s\left(\frac{1}{r+c_{1} \cos \left[k\left(\frac{1}{2}-n\right)\right]}-\frac{1}{r+c_{1} \cos \left[k\left(\frac{1}{2}+n\right)\right]}\right)
$$

where $r=q \cosh (\delta t)$ and $s=\delta q \sinh (\delta t)$. This clearly equals $-M_{-n}$, while $M_{0}=0$. Hence, using Eq. (26), $M=0$.

\section{MAXIMUM AMPLITUDES}

Now, the question is what absolute maximum amplitude the breather, Eq. (21), can reach for any particular value of $\kappa$. From Eq. (21), we can find

$$
\left|\Psi_{n}(t)\right|_{\max }=\frac{2\left(1+q^{2}\right) v}{q-c_{1}}-q
$$




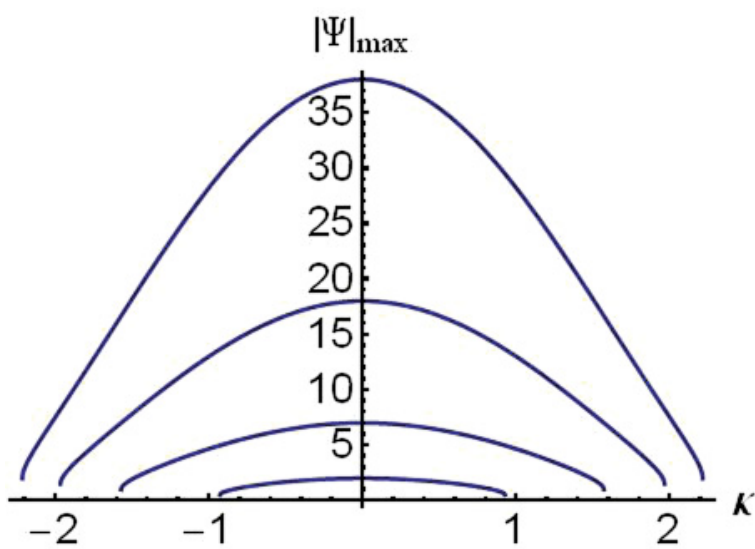

FIG. 3. (Color online) Maximum value of field defined by Eq. (28). From upper to lower, the curves are for $q=2,1.5,1$, and 0.5 , respectively.

or, in terms of $\kappa$,

$$
\begin{aligned}
\left|\Psi_{n}(t)\right|_{\max }= & q\left[q^{2}+\left(1+q^{2}\right) \cos (\kappa)\right]+\sqrt{2}\left(1+q^{2}\right) \cos (\kappa / 2) \\
& \times \sqrt{\left(1+q^{2}\right) \cos (\kappa)-1+q^{2}} .
\end{aligned}
$$

We present the dependence of the maximum amplitude on $\kappa$ in Fig. 3. For any given $q$, we can expect the highest amplitude when $\kappa \rightarrow 0$ while the maximum is reduced to the background value $q$ at the edges of the instability interval. At the point of maximum growth rate, $\kappa_{0}=\arccos \left(\frac{1}{1+q^{2}}\right)$, the maximum amplitude is given by

$$
\left|\Psi_{n}\right|_{\max }=q \sqrt{q^{2}+1}\left(\sqrt{q^{2}+1}+\sqrt{q^{2}+2}\right) .
$$

It depends only on $q$. If, additionally, $q=1$, then $\left|\Psi_{n}\right|_{\max }=$ $2+\sqrt{6}$.

The maximum value of $\Psi_{n}$ is of great interest in potential applications of these results, since this type of solution for the continuous NLSE has been suggested as a prototype for rogue waves in a number of publications [5-9]. Rogue waves can also appear in discrete systems [23]. Thus, from this point of view, the particular solution with $\kappa=0$, where the period along the $n$ axis tends to infinity, deserves special consideration. This will be done in Sec. X.

Despite the close analogy with the NLSE AB solutions, there are also significant differences. One of them is the fact that the background amplitude $q$ could be eliminated in the NLSE case, but not here. Indeed, for NLSE, there is the so-called scaling transformation (see Eq. (2.3) of Ref. [20]) that allows us to transform any solution with a given amplitude to one with unit amplitude. The reverse also holds: A solution with amplitude one can be generalized to a solution with an arbitrary amplitude. In particular, the background value $q$ in the case of the NLSE can always be transformed to the unit background case. This operation will rescale the other variables as well. There is no similar transformation in the case of A-L equation. Thus, we have to keep the parameter $q$ in the solution, Eq. (22), as an independent parameter that controls the value of the background in the solution.

Another significant difference is that the discrete variable $n$ can be shifted by any real constant $n_{0}$, i.e., we can replace $n$ by $n-n_{0}$ in all equations above. If $n_{0}$ is an integer, the solution will be identical to the original solution but shifted along the $n$ axis. On the other hand, if $n_{0}$ is not an integer, then the solution, which is defined only at discrete sites, will be different. We recall that, in the NLSE case, translations along the transverse variable lead to a solution which has the same profile but is just shifted. We present the solution in Fig. 2 as being continuous, but we need to remember that it is defined only at integer values of $n$. The grid lines here correspond to $n=0, \pm 1, \pm 2, \ldots$.

\section{EVOLUTION OF SPECTRAL COMPONENTS}

In many practical applications, we are interested in the spectra of the field distribution. Making a theoretical study of them provides us with more insight into the physical system itself. To study the spectral components of the field, we can take a discrete Fourier transform of $N$ points along the $n$ axis for any $t$, using the definition

$$
v_{k}(t)=\frac{1}{N} \sum_{n=0}^{N-1} \Psi_{n}(t) \exp (-2 \pi i n k / N) .
$$

Clearly, $v_{k+N}(t)=v_{k}(t)$. The period of the solution is $T=$ $2 \pi / \kappa$. We mostly take this to be an integer and so we can set $N=T$. If $T$ is not an integer, then it has to be sufficiently large to get a good approximation of the spectral components. Ideally, we should consider the limit of infinite $N$, thus obtaining an infinite number of spectral components. However, if $T$ is an integer, then it is sufficient to take a sum over the period $T$. The number of spectral components then is finite.

We note that the spectral components of a discrete chain are basically the normal modes of the system under consideration. Generally, the number of normal modes is infinite for a chain with an infinite number of oscillators. However, if the period of excitation is an integer, the number of normal modes is finite, since all higher-order ones are the same as those in the set of the finite number of the lowest-order modes. Thus, by controlling the parameter $\kappa$, within the infinite dimensional dynamical system, we can isolate finite-dimensional sub-systems.

Let us consider the spectra for the solution in simplest form, Eq. (22), which is shown in Fig. 2. For the maximum growth case, where $q=1$ and $\kappa=\pi / 3$, the transverse period $T=2 \pi / \kappa=6$, i.e., it is an integer. For this case, the highest harmonic (or normal mode) is $k=3$. To start with, we take zero offset, $n_{0}=0$. All four components can be calculated analytically:

$$
\begin{gathered}
\left|v_{0}(t)\right|^{2}=\left(\frac{4}{D}\right)^{2}\left[(1+3 \cosh (4 t))^{2}+81 \sinh ^{2}(8 t)\right], \\
\left|v_{1}(t)\right|^{2}=\left|v_{-1}(t)\right|^{2}=\frac{24}{D^{2}} \cosh (4 t)[1+3 \cosh (4 t)]^{2}, \\
\left|v_{2}(t)\right|^{2}=\left|v_{-2}(t)\right|^{2}=\left(\frac{12}{D}\right)^{2} \cosh ^{2}(2 t) \cosh (4 t), \\
\left|v_{3}(t)\right|^{2}=\left|v_{-3}(t)\right|^{2}=\frac{96}{D^{2}} \cosh (4 t) .
\end{gathered}
$$

where $D=5+6 \cosh (4 t)+9 \cosh (8 t)$. Clearly, we have $v_{-k}(t)=e^{i k \kappa} v_{k}(t)$ so that $\left|v_{-k}(t)\right|=\left|v_{k}(t)\right|$.

The $t$ evolution of these spectral components is shown in Fig. 4. The fundamental component, $v_{0}$, initially (at $t \rightarrow-\infty$ ) 


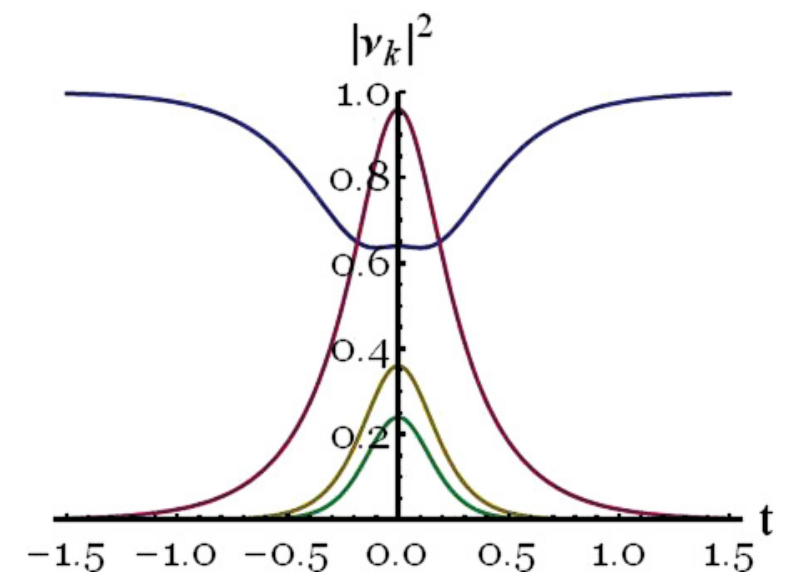

FIG. 4. (Color online) Evolution of the components of the discrete Fourier transform of the breather solution given by Eq. (22) for $n_{0}=$ 0 . Here, $q=1$ and $\kappa=\pi / 3$. The fundamental spectral component $\left(\left|v_{0}\right|^{2}\right)$ approaches 1 for large $|t|$ and is in blue, while the other three curves show successively the components $\left|v_{1}\right|^{2}$ (magenta, upper), $\left|v_{2}\right|^{2}$ (olive, mid), and $\left|v_{3}\right|^{2}$ (green, lower). Thus, the amplitudes and their maxima decrease as $k$ increases from 1 to 3. See Fig. 3.7 of Ref. [20] for the analogous spectra of AB solutions of the NLSE.

contains all the energy $\left(\sim|\nu|^{2}\right)$, and other components have none. At the point of the maximal development of $\mathrm{MI}$ $(t=0)$, this fundamental component is depleted and reaches its minimum, while other components gain a considerable fraction of the energy, as can be seen in Fig. 4. After the evolution is completed (at $t \rightarrow+\infty$ ), all the energy returns to the central component.

The moduli of the spectral components (not squared) at the point of maximum modulation, $t=0$, are shown in Fig. 5. The nearest sidebands, $\left|v_{ \pm 1}\right|$, at this point have the highest amplitude, while the amplitudes of higher components decrease with $k$. This distribution is also similar to the spectra of AB solutions of the NLSE case, despite the fact that here we are dealing with a finite number of components.

The spectra are symmetric when the offset $n_{0}$ is either an integer or half integer. It turns out that we get results of the simplest form for the latter case, when the offset $n_{0}=1 / 2$. Then the number of spectral components is reduced by one so there are only three independent values of $\Psi_{n}$. Consequently, we

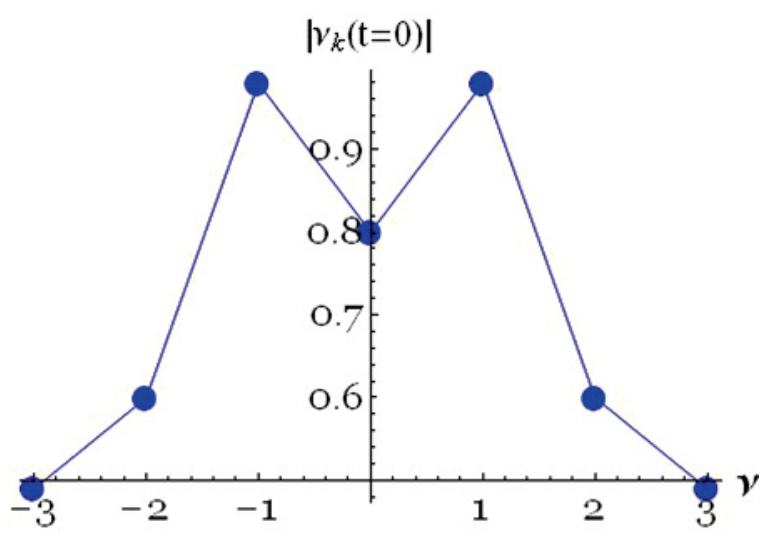

FIG. 5. (Color online) Lowest-order spectral components $\left|v_{k}\right|$ shown by thick blue dots at $t=0$ for $n_{0}=0$.

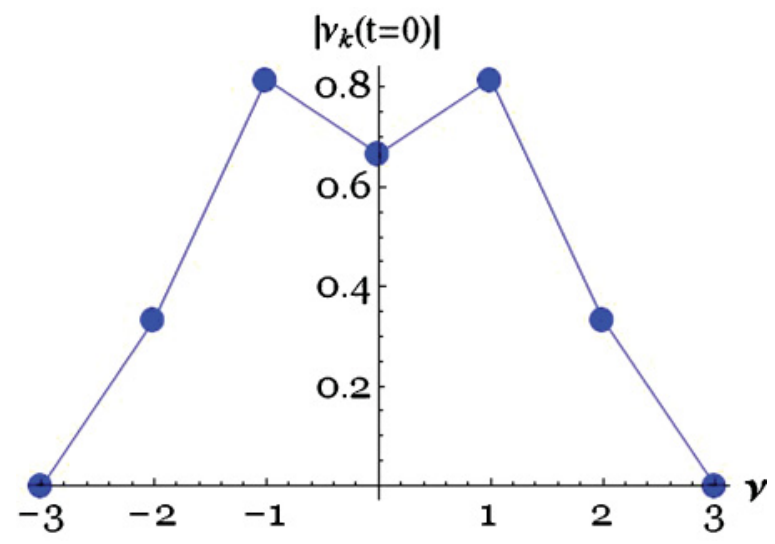

FIG. 6. (Color online) Lowest-order spectral components $\left|v_{k}\right|$ shown by thick blue dots at $t=0$ for $n_{0}=1 / 2$.

have only three independent spectral components. Calculating the squared moduli of these components, we find

$$
\begin{gathered}
\left|v_{0}(t)\right|^{2}=\frac{1}{9}\left[9-\operatorname{sech}^{2}(4 t)-4 \operatorname{sech}(4 t)\right], \\
\left|v_{1}(t)\right|^{2}=\left|v_{-1}(t)\right|^{2}=\frac{2}{3} \operatorname{sech}(4 t) \\
\left|v_{2}(t)\right|^{2}=\left|v_{-2}(t)\right|^{2}=\frac{1}{4} \operatorname{sech}^{2}(2 t) \operatorname{sech}(4 t) . \\
\left|v_{3}(t)\right|^{2}=\left|v_{-3}(t)\right|^{2}=0 .
\end{gathered}
$$

These components at $t=0$ are shown in Fig. 6. Qualitatively, the plot is similar to the distribution of components in Fig. 5, except for the fact that the components $\left|v_{ \pm 3}\right|$ are zero.

Since there are only a few components $(N=6)$, it is easy to verify Parseval's theorem here:

$$
A(t)=\frac{1}{N} \sum_{n=0}^{N-1}\left|\Psi_{n}(t)\right|^{2}=\sum_{k=0}^{N-1}\left|v_{k}(t)\right|^{2} .
$$

Interestingly, the "total energy" defined as $Q=\sum Q_{n}$, where $Q_{n}=\frac{1}{2}\left(\psi_{n} \psi_{n+1}^{*}+\psi_{n}^{*} \psi_{n+1}\right)-q^{2}$, and the summation is over the period $T$, is independent of $t$, i.e., it is conserved, while the function $A(t)$ is not. In fact, for $n_{0}=1 / 2$,

$$
A(t)=\left[1-\frac{1}{3} \operatorname{sech}^{2}(2 t)+\frac{4}{3} \operatorname{sech}(4 t)\right] .
$$

A qualitatively similar evolution pattern for spectral components can be obtained for the general case, Eq. (21). One example is shown in Fig. 7. Here, the number of independent spectral components is seven and their distribution is close to being triangular. We note here that triangular spectra are distinctive features of processes that involve modulation instability (see Fig. 5(b) of Ref. [24]). For periods $T$ that are not integers, we have to consider an infinite number of spectral components. In this instance, their evolution is very similar to that of the AB solution of the continuous NLSE [17].

\section{FPU RECURRENCE}

As we have seen in the previous section, the breather solution has a unique property. Namely, the process defined by this solution starts with all the energy in a single normal mode of the system. The energy is distributed between other spectral components in the middle of the process, at $t=0$. Finally, all 


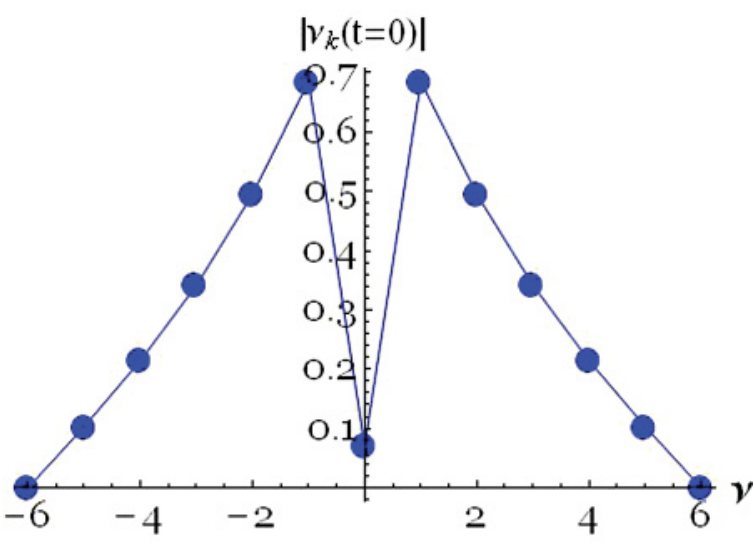

FIG. 7. (Color online) Lowest-order spectral components $\left|v_{k}\right|$ shown by thick blue dots at $t=0$ when $\kappa=\pi / 6, q=1$ and $n_{0}=$ $1 / 2$. The period $T$ in this case occupies 12 sites.

the energy is returns to the initial central mode. This property is presently known as "recurrence."

This is exactly what occurred in the numerical simulations of Fermi, Pasta, and Ulam [15] for a fixed chain of 64 oscillators. At that time (1954), this feature of the nonlinear chain was viewed as a paradox, since the authors expected equipartition of energy between the modes, rather than its return to the initial mode. Thus, our results provide a compete solution of the FPU paradox for a system defined by the A-L equation. Indeed, the system is discrete, just as in the original FPU problem. Moreover, as we discussed above, it can be reduced to a finite dimensional one. Our equations allow us to choose any number of oscillators. The recurrence was a surprise at the time of the first studies [15] but seems to be natural behavior for nonlinear systems such as the NLSE $[25,26]$.

Various models admit FPU recurrence [27,28]. The time evolution of normal modes for them is similar to those in Fig. 4. However, in the case of nonintegrable models, the recurrence is always approximate, while for integrable models such as A-L equation, the recurrence is exact. Moreover, the spectral components that are basically normal modes of the system can be expressed in analytical form and this possibility is a major step forward in solving the FPU paradox.

A closer investigation of the recurrence feature of the solution, based on the trajectories of the solution on the complex plane, can be made. Let us analyze a particular case, Eq. (22), presented in Fig. 2. Figure 8 shows the evolution of the complex amplitude $\Psi_{n}(t)$ of the field in time at the site $n=0$. To be definite, we have chosen the phase $\phi=\pi / 2$. This corresponds to the initial point $-i q$ on the complex plane of $\Psi_{n}$. At $t=-\infty$, the trajectory starts at this saddle point, makes a full loop (as shown by the green curve), and returns to another saddle point, located at $+i q$. The shorter (blue) curve, with arrows connecting the two saddle points, corresponds to $\Psi_{n}(t)$ in between the two maxima, e.g., at $n=3$.

The curves for other values of $n$ are also ellipses (not shown) that connect the two saddle points. We can notice that the two sets of trajectories in Fig. 8 are symmetric. The upper point $+i q$ can equally well be the starting point of evolution, which then ends at the lower point $-i q$. This is a consequence of the fact that any point on a circle of radius $q$ is a saddle, and the whole complex plane can be rotated by any angle $\phi$.

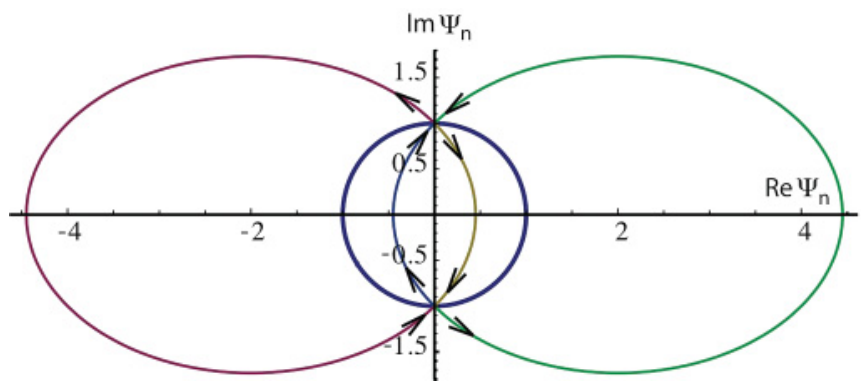

FIG. 8. (Color online) Trajectories on the complex plane defined by the solution, Eq. (22), for $q=1$ and $\kappa=\arccos \left(\frac{1}{1+q^{2}}\right)=\pi / 3$, $n=0$, and $n= \pm 3$. The solid (blue) circle around the origin is the locus of starting points of the breather solution with phase $\phi$. When $\phi= \pm \pi / 2$, the upper and lower points on this circle are the starting and final saddle points of the modulation instability. This figure shows clearly the nonlinear phase shift $\pi$ that the $C B$ wave has gained when the breather solution evolves from $t=-\infty$ to $t=\infty$.

Thus, generally speaking, the trajectories shown in Fig. 8 are heteroclinic orbits, since they connect two different saddle points in the phase space.

To conclude this section, let us show analytically that all trajectories in complex plane are ellipses. In fact, for $q=1$ and $\kappa=\pi / 3$ (and $n_{0}=0$ ), the real and imaginary parts for all $n$ are related as follows:

$$
\begin{gathered}
{\left[5 R_{n}(t)-1\right]^{2}+5 J_{n}^{2}(t)=6, \quad n=1,2,4,5,7, \ldots,} \\
{\left[R_{n}(t)-2\right]^{2}+2 J_{n}^{2}(t)=6, \quad n=0,3,6, \ldots}
\end{gathered}
$$

Clearly, these are equations defining ellipses in the complex plane.

If we retain $\kappa=\pi / 3$ so that the period is still $T=6$, then we can generalize these equations to arbitrary $q$, again taking $n_{0}=0$

$$
\begin{aligned}
& {\left[\left(1+9 q^{2}\right) R_{n}(t)+q\left(3 q^{2}-5\right)\right]^{2}} \\
& \quad+\left(1+q^{2}\right)\left(1+9 q^{2}\right) J_{n}^{2}(t)=F,
\end{aligned}
$$

for $n=1,2,4,5,7, \ldots$, while

$$
\left[2 R_{n}(t)-q\left(1+3 q^{2}\right)\right]^{2}+4\left(1+q^{2}\right) J_{n}^{2}(t)=F,
$$

for $n=0,3,6, \ldots$, where $F=3\left(3 q^{2}-1\right)\left(1+q^{2}\right)^{2}$. From Eq. (4), we need $q>1 / \sqrt{3}$.

For offset $n_{0}=1 / 2$, the results are even simpler:

$$
\left[R_{n}(t)-q\right]^{2}+J_{n}^{2}(t)=3 q^{2}-1, \quad n=0,1,3,4,6, \ldots,
$$

so the trajectories lie on a circle of radius $\sqrt{3 q^{2}-1}$ with its center at $(q, 0)$. Again, $q>1 / \sqrt{3}$. For $n=2,5, \ldots$, we have $R_{n}(t)=\frac{1-q^{2}}{2 q}$, which is a constant, indicating that the trajectory is just a vertical line.

\section{NONLINEAR PHASE SHIFT OF THE BACKGROUND}

As we have seen, the description using trajectories in the complex plane also demonstrates the recurrence. The $\mathrm{CB}$ solution at the lower point $-i q$ is transformed to the upper point $+i q$ on the circle which is also a CB solution, but with shifted phase. In the case with the maximum growth rate, the shift is $\pi$. Thus, after the breather has developed, the plane 


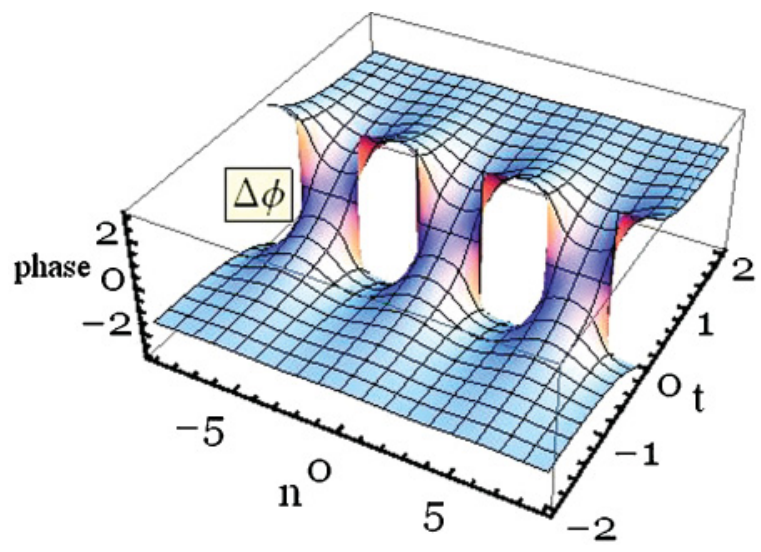

FIG. 9. (Color online) Phase diagram $\operatorname{Arg}\left[\Psi_{n}(t)\right]$ for MI solution defined by Eq. (22). Here, $q=1$ and $\kappa=\pi / 3$. The development of the breather causes a nonlinear phase shift $\Delta \phi$ to the background. In the case of the maximum growth rate, $\Delta \phi$ is equal to $\pi$.

wave has gained a phase shift of $\pi$. This is a phase shift that the initial state has suffered as a result of a nonlinear process on top of a CB wave.

The nonlinear phase shift (NPS) depends on the spatial frequency, $\kappa$. It is zero at the upper limit of instability and increases to $2 \pi$ as $\kappa \rightarrow 0$. Generally, trajectories in Fig. 8, defined by Eq. (22), connect two saddle points, which have a specific phase difference between them, on a circle at the complex plane. The starting point is defined by the arbitrary phase $\phi$. The final point of the evolution gains an additional phase,

$$
\Delta \phi=2 \arccos \left[2 \frac{1+q^{2}}{q^{2}} \sin ^{2}\left(\frac{\kappa}{2}\right)-1\right],
$$

which depends on $q$ and $\kappa$. Hence, the total phase when $t \rightarrow \infty$ will be $\phi+\Delta \phi$. In other words, the initial CB wave is transformed from $\Psi=-q \exp (i \phi)$ into

$$
\Psi=-q \exp (i \phi+i \Delta \phi),
$$

with an additional phase $\Delta \phi$.

This transformation is illustrated in Fig. 9. The figure shows evolution of the phase of the solution in $t$ defined as $\operatorname{Arg}\left[\Psi_{n}(t)\right]$ for the whole breather solution, Eq. (22). The resulting phase after the breather has evolved and disappeared is clearly different from the phase of initial CB solution.

Thus, in absence of any perturbation, the CB wave would stay unperturbed, while in presence of the breather, it gains an additional phase shift. The phase shift gained after the breather with the maximum growth rate is equal to $\pi$. Otherwise, it varies from 0 to $2 \pi$ within the instability band as shown in Fig. 10.

This is a remarkable result. To some extent, this phase shift is analogous to the Berry phase [16,29] gained by quantum states and other physical systems after a certain sequence of processes which involve the system. However, we should remember that the Berry phase is essentially a linear effect. For breather solutions of the NLSE and A-L equations, the phase shift is nonlinear. This is an example of a carrier phase shift caused by the wave envelope being involved in a nonlinear process. Thus, we call it the "nonlinear phase shift" (NPS).

The NPS is an additive phenomenon. When two breathers are excited simultaneously or one after the other, the total

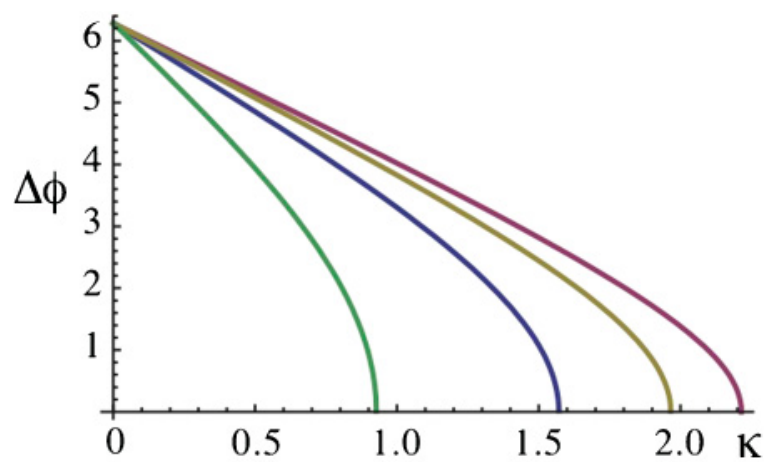

FIG. 10. (Color online) Nonlinear phase shift (NPS) $\Delta \phi$ vs $\kappa$. From upper to lower, the curves are calculated for $q=2,1.5,1$, and 0.5 , respectively.

phase shift of the background is equal to the sum of the phase shifts caused by each of the breathers. This is similar to the phase shifts generated by ABs of the NLSE (see Fig. 3.19 of Ref. [20]). The largest phase shift of $2 \pi$ is expected when $\kappa \rightarrow 0$ (see Fig. 10). Clearly, this is a case that deserves special consideration.

\section{FUNDAMENTAL ROGUE WAVE}

The long-period limit of the solution, Eq. (21), can be obtained by taking the parameter $v$ (or $\kappa$ ) to be small. The infinite period limit of the periodic solution means that $|\Psi|$ has only one peak instead of a periodic pattern along the $n$ axis. In this case we find that the growth rate,

$$
\delta \rightarrow 2 q \sqrt{1+q^{2}} \kappa=4 q \sqrt{v\left(1+q^{2}\right)},
$$

tends to zero. Choosing the minus sign for $c_{1}$ and expanding terms to order $v$, i.e., $\kappa^{2}$, we obtain the rational solution (see Eq. (34) below) which depends on the ratio of two polynomials.

Instead of considering a limit, we can also derive the rational solution directly using our linear ansatz. In order to do this, we note that Eq. (19) simplifies greatly if the last term in it is zero, i.e., if $m^{\prime}(t)=4 q^{2}$. The slope $m(t)$ is then given by the linear function of $t$ :

$$
m(t)=4 q^{2} t
$$

Now Eq. (19) is

$$
\frac{d P_{n}}{d t}=8 q^{3} t
$$

It has a solution

$$
P_{n}(t)=4 q^{3} t^{2}+k(n) .
$$

The simplest form for $k(n)$ is to try $k(n)=s+p n^{2}$ with unknown coefficients $s$ and $p$. On substituting into the real part of the expansion, Eq. (16), we find that $s=1 /\left[4 q\left(1+q^{2}\right)\right]$ and $p=q /\left(1+q^{2}\right)$, so that

$$
R_{n}(t)+q=\frac{1}{P_{n}(t)}=\frac{4 q\left(1+q^{2}\right)}{1+4 n^{2} q^{2}+16 q^{4}\left(1+q^{2}\right) t^{2}},
$$

Thus

$$
\Psi_{n}(t)=\left[\frac{4 q\left(1+q^{2}\right)\left(1+4 i q^{2} t\right)}{1+4 q^{2} n^{2}+16 q^{4}\left(1+q^{2}\right) t^{2}}-q\right] e^{i \phi},
$$




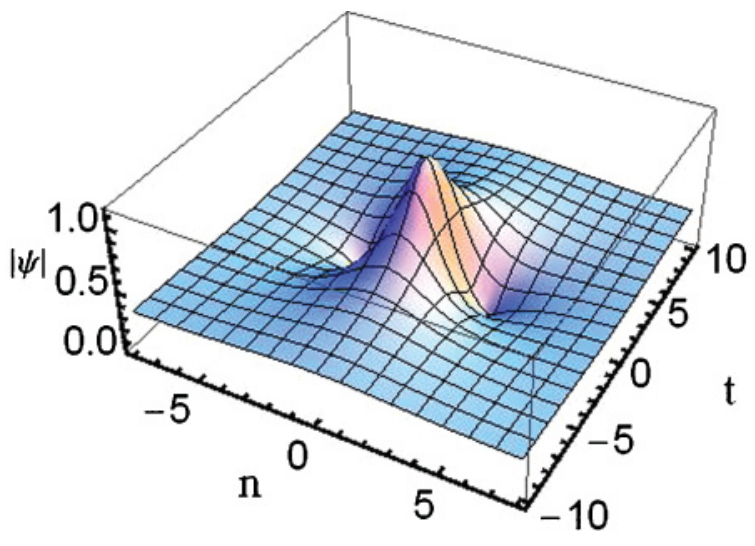

FIG. 11. (Color online) Rational solution of the first order defined by Eq. (34). Here, $q=1 / 3$.

which is indeed a solution of the A-L equation. This result, Eq. (34), can be called the first-order $(j=1)$ rational or fundamental rogue wave solution. It is shown in Fig. 11. The solution is localized in both $n$ and $t$ directions.

This solution is analogous to the Peregrine solution of the NLSE (see Ref. [4] and Eq. (3.47) in the book [20]). Although the structure of the solution is basically the same as for the NLSE, the $q$ dependence is far from being trivial. Thus, deriving this solution is not a straightforward exercise, even when a similar solution of the NLSE is known. This remark can be related to all exact solutions of the A-L equation.

The peak amplitude of the solution is $|\Psi|_{\max }=q\left(3+4 q^{2}\right)$. Instead of exponential growth, this solution increases in $t$ only algebraically (since $\delta \rightarrow 0$ ). In fact, $\psi \sim-q+\frac{i}{q t}$ for large $|t|$.

The solution can also be written in a more general form:

$$
\Psi_{n}(t)=q\left[4\left(1+q^{2}\right) \frac{G_{n}(t)+4 i q^{2} t H_{n}(t)}{D_{n}(t)}-1\right] e^{i \phi},
$$

where $G_{n}(t)=1, H_{n}(t)=1$, and $D_{n}(t)=1+N^{2}+T^{2}$ with scaling factors $N(n)=2 q n$ and $T(t)=4 q^{2} \sqrt{1+q^{2}} t$. This form is convenient for writing higher-order rational solutions $\Psi_{n}(t)$ [14].

The phase diagram for the solution, Eq. (34), is shown in Fig. 12. The phase of the CB has a phase jump $2 \pi$, in contrast

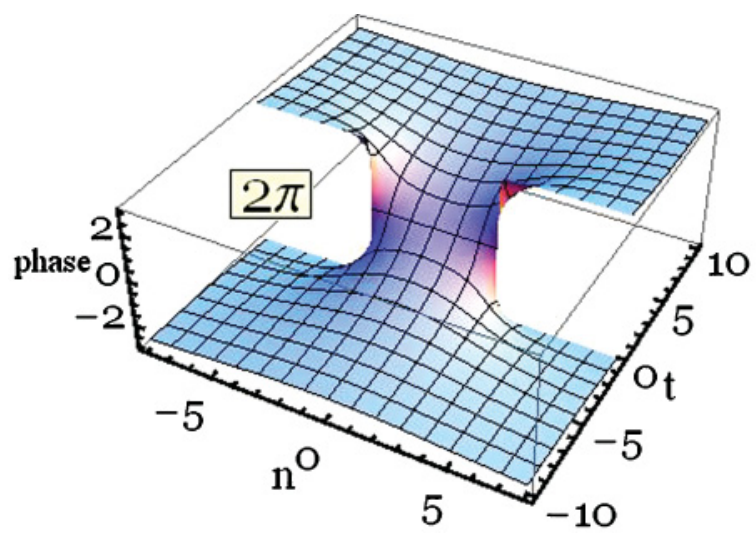

FIG. 12. (Color online) Phase diagram $\operatorname{Arg}\left[\Psi_{n}(t)\right]$ for the firstorder rogue wave solution defined by Eq. (34). Here, $q=1 / 3$. This nonlinear event causes a phase addition of $2 \pi$ across the rogue wave. to the case considered above. Although this NPS seems to be equivalent to a zero shift, we cannot ignore the continuous evolution through all the stages of phase change at the center of the disturbance. If any carrier wave is involved in the evolution, as happens in most practical problems, the phase shift of $2 \pi$ occurring within a short distance in the middle of a rogue wave may dramatically change the overall dynamics. Thus, the NPS considered here can influence events involving rogue waves in this unusual way. Higher-order rational solutions [14] have nonlinear phase shifts which are multiples of $2 \pi$, thus significantly complicating the processes related to them.

\section{CONCLUSION}

We have studied modulation instability of discrete systems described by the Ablowitz-Ladik equation and given exact solutions that are a direct continuation of this instability at larger amplitudes. We emphasize that our solutions do not involve approximate functions which merely resemble the actual ones. Exact solutions are similar to those derived previously for the NLSE [17,21], although direct discretization of the continuous breather solutions seems to be highly nontrivial. These solutions are of great importance for mathematical physics as they represent a complete solution of the FermiPasta-Ulam paradox of recurrence in discrete systems modeled by the A-L equation: Namely, the nonlinear discrete system that starts the evolution in one of the normal modes, distributes the initial energy between other normal modes, but returns it to the original mode when the evolution is completed.

Another remarkable property of the recurrence is the nonlinear phase shift (NPS) that the initial mode gains as a result of the nonlinear process. The NPS is the difference between the phases of the normal mode after and before the event. It depends on the spatial frequency of the initial perturbation and varies from 0 to $2 \pi$ within the instability band. The NPS can be a property of a wide variety of nonlinear processes when the evolution starts in a single normal mode of a system. This phenomenon deserves further studies, as it can influence the carrier waves in optics, ocean waves, and other branches of physics.

Finally, we also derived a rogue wave solution of the A-L equation and showed that it is a particular case of the family of breather solutions when the spatial period of the initial perturbation becomes infinite. This solution is localized both in space and time and represents a unique event that "appears from nowhere and disappears without a trace" [30,31].

One of the possible applications of the results that we obtained in this work is to arrays of optical waveguides [23]. Although the equations governing the latter system are slightly different from the A-L equations, qualitatively similar effects are still observable. Clearly, one of these effects is the rogue wave [23].

\section{ACKNOWLEDGMENTS}

The authors acknowledge the support of the A.R.C. (Discovery Project DP110102068). One of the authors (N.A.) is a grateful recipient of support from the Alexander von Humboldt Foundation (Germany). 
[1] V. I. Bespalov and V. I. Talanov, Zh. Eksp. Teor. Fiz., Pis'ma Red. 3, 471 (1966) [JETP Lett. 3, 307 (1966)].

[2] T. B. Benjamin and J. E. Feir, J. Fluid Mech. 27, 417 (1967).

[3] A. M. Turing, Philos. Trans. R. Soc. London B 237, 37 (1952).

[4] D. H. Peregrine, J. Australian Math. Soc., Ser. B 25, 16 (1983).

[5] K. B. Dysthe and K. Trulsen, Phys. Scr., T 82, 48 (1999).

[6] V. V. Voronovich, V. I. Shrira, and G. Thomas, J. Fluid Mech. 604, 263 (2008).

[7] I. Ten and H. Tomita, Reports of RIAM Symposium No. 17SP1-2, Proceedings of a symposium held at Chikushi Campus, Kyushu University, Kasuga, Fukuoka, Japan, March 10-11, 2006 (unpublished).

[8] D. Clamond, M. Francius, J. Grue, and C. Kharif, Eur. J. Mech. B 25, 536 (2006).

[9] V. I. Shrira and V. V. Geogjaev, J. Eng. Math. 67, 11 (2010).

[10] A. Mohamadou, F. Fopa, and T. C. Kofané, Opt. Commun. 266, 648 (2006).

[11] P. Marquie, J. M. Bilbault, and M. Remoissenet, Phys. Rev. E 51, 6127 (1995).

[12] J. M. Bilbault and P. Marquie, Phys. Rev. E 53, 5403 (1996).

[13] D. Hennig and G. P. Tsironis, Phys. Rep. 307, 333 (1999).

[14] A. Ankiewicz, N. Akhmediev, and J. M. Soto-Crespo, Phys. Rev. E 82, 026602 (2010).

[15] E. Fermi, J. Pasta, and S. Ulam, Los Alamos Report No. LA1940, 1955 [reprinted in E. Fermi, Collected Papers, edited by E. Segré (University of Chicago Press, Chicago, 1965), Vol. II, p. 978.]
[16] M. Berry, Proc. R. Soc. London, Ser. A 392, 45 (1984).

[17] N. Akhmediev and V. I. Korneev, Theor. Math. Phys. (USSR) 69, 189 (1986) [69, 1089 (1986)].

[18] M. J. Ablowitz and J. F. Ladik, Stud. Appl. Math. 55, 213 (1976).

[19] M. J. Ablowitz and J. F. Ladik, J. Math. Phys 17, 1011 (1976).

[20] N. Akhmediev and A. Ankiewicz, Solitons, Nonlinear Pulses and Beams (Chapman and Hall, London, 1997).

[21] N. Akhmediev, V. M. Eleonckii, and N. E. Kulagin, Theor. Math. Phys. (USSR) 72, 809 (1987) [72, 183 (1987)].

[22] K. Narita, J. Phys. Soc. Jpn. 59, 3528 (1990); 60, 1497 (1991).

[23] Yu. V. Bludov, V. V. Konotop, and N. Akhmediev, Opt. Lett. 34, 3015 (2009).

[24] J. M. Dudley, G. Genty, F. Dias, B. Kibler, and N. Akhmediev, Opt. Express 17, 21497 (2010).

[25] G. Van Simaeys, Ph. Emplit, and M. Haelterman, Phys. Rev. Lett. 87, 033902 (2001).

[26] N. Akhmediev, Nature (London) 413, 267 (2001).

[27] R. L. Bivins, N. Metropolis, and J. Pasta, J. Comput. Phys. 12, 65 (1973).

[28] K. Yoshimura, Phys. Rev. E 62, 6447 (2000).

[29] F. Wassmann and A. Ankiewicz, Appl. Opt. 37, 3902 (1998).

[30] N. Akhmediev, A. Ankiewicz, and M. Taki, Phys. Lett. A 373, 675 (2009).

[31] N. Akhmediev, J. M. Soto-Crespo, and A. Ankiewicz, Phys. Lett. A 373, 2137 (2009). 